\title{
METAPHASE I BOUND ARMS AND CROSSING OVER FREQUENCY \\ IN RYE III. NON-CHIASMATE BONDS IN DESYNAPTIC PLANTS
}

\author{
J. DRELLANA AND R. GIRALDEZ*
}

Departamento de Genética, Facultad de Biologia, Universidad Complutense, Madrid, Spain; *Departamento de Genética, Facultad de Biologla, Universidad de Oviedo, Oviedo, Spain

Received 4.ii.83

\section{SUMMARY}

\begin{abstract}
In order to analyze the correspondence between the number of metaphase I bonds and the chiasma frequency of specific chromosome arms in desynaptic plants, two F1 hybrids were backcrossed to the parental inbred lines. The plants obtained were desynaptic. Some of these plants were heterozygous for a prominent telomeric C-band in chromosomes $1 \mathrm{R}$ (long arm) and/or 2R (short arm). From the frequency of bound arms at metaphase I and the frequency of recombined chromosomes at anaphase $I$ in the arms considered, estimates of chiasma frequencies have been derived. The results have been compared with those obtained for the F1s between inbred lines from which the desynaptic plants were produced (Giraldez and Orellana, 1979; Orellana and Giraldez, 1981) as well as with those obtained in an open pollinated variety of rye (Orellana and Giraldez, 1981). The excess of anaphase I parental chromosomes found in the desynaptic plants can be explained on the assumption that some of the bonds appearing at metaphase I are achiasmate and a remnant of prophase pairing.
\end{abstract}

\section{INTRODUCTION}

In previous studies (Giraldez and Orellana, 1979; Orellana and Giraldez, 1981) a procedure was used for estimating chiasma frequency in specific chromosome arms of rye marked by Giemsa banding in meiosis. Plants differing in C-banding pattern of specific chromosome arms were used. From the frequency of being bound at metaphase I and the frequency of recombinant chromosomes at anaphase I and metaphase II in the arms considered, estimates of actual chiasma frequencies were derived.

In those instances the materials employed were F1s between inbred lines and an open pollinated variety of rye. The results indicated that the numbers of metaphase I bonds and the frequency of anaphase I recombinant chromosomes were not very different in the short arm of chromosome $2 \mathrm{R}$ but differed in the long arm of chromosome $1 R$, especially in one of the $F 1 s$ between inbred lines and in the open pollinated variety analyzed. The excess of anaphase I parental chromosomes found in these cases was interpreted as resulting from the formation of more than one chiasma per bond in these chromosome arms.

In the present study, the comparison between metaphase I bonds and chiasma frequency has been extended to partially desynaptic inbred plants of rye in which a lower chiasma frequency is expected. On the other hand, in desynaptic plants of rye a loss of bonds is produced during metaphase 
I which leads to the appearance of univalents at this stage (Orellana et al., unpublished). The material is heterozygous for $\mathrm{C}$-bands and thus suitable for the analysis of the nature of metaphase I bonds in desynaptic plants and the possible mechanism by which bound arm loss takes place.

\section{MAterials AND Methods}

In the material analyzed, three inbred lines of rye were involved, P, M and E. Two F1s (F1PE and F1PM) obtained from crosses between these three lines were backcrossed by their parental inbred lines and their offspring (B1PEE, B1PMM and B1PMP) formed the material for this study. As described earlier (Giraldez et al., 1979) the inbred lines used differed in the presence $(+)$ or absence $(-)$ of telomeric $C$-heterochromatin in the long arm of chromosome $1 \mathrm{R}$ and in the short arm of chromosome $2 \mathrm{R}$. In the following scheme, the crosses performed and the C-heterochromatin constitution of chromosome arms $1 \mathrm{R}$ long and $2 \mathrm{R}$ short in the plants obtained are indicated:

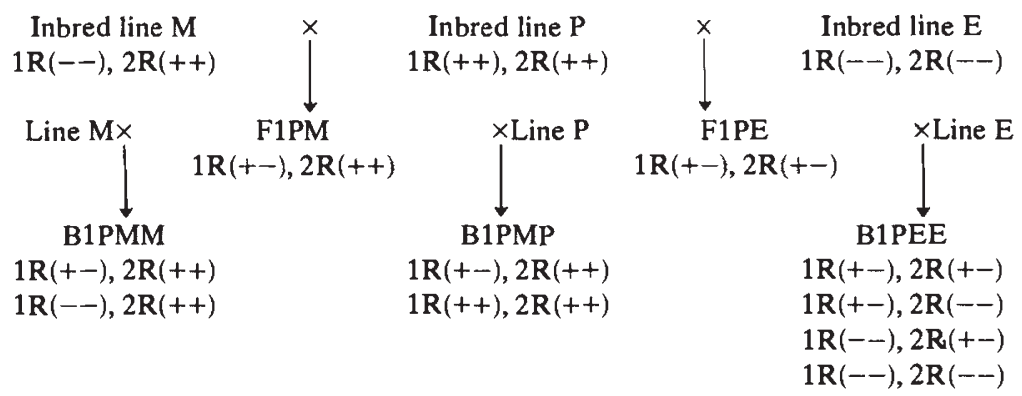

In order to obtain mitotic metaphase cells, seeds were germinated on wet filter paper in Petri dishes at room temperature. When primary roots were $1-2 \mathrm{~cm}$ long they were excised and immersed in tap water at $0^{\circ} \mathrm{C}$ for $48 \mathrm{~h}$ to shorten the chromosomes. Subsequently the tips were fixed in acetic alcohol $1: 3$. For meiotic cells, anthers were fixed in acetic alcohol $1: 3$. The fixed material was squashed and stained following the Giemsa Cbanding technique described previously (Giraldez and Orellana, 1979).

The chromosome nomenclature used was the one proposed at the workshop on rye chromosome nomenclature and homoeology relationships held at Wageningen, The Netherlands (Sybenga, 1982). The tentative correspondence between this nomenclature and the one used in previous work (Giraldez et al., 1979) is:

Present nomenclature

$\begin{array}{rrrrrrr}1 \mathrm{R} & 2 \mathrm{R} & 3 \mathrm{R} & 4 \mathrm{R} & 5 \mathrm{R} & 6 \mathrm{R} & 7 \mathrm{R} \\ 7 & 3 & 2 & 4 & 6 & 5 & 1\end{array}$

Giraldez et al. 1979

\section{Results}

Using the C-banding technique it was possible to identify chromosomes $1 R, 2 R$ and $5 R$ in meiotic cells of all plants analyzed. Figs. 1 and 2 show examples of metaphase-anaphase I C-banded cells. 


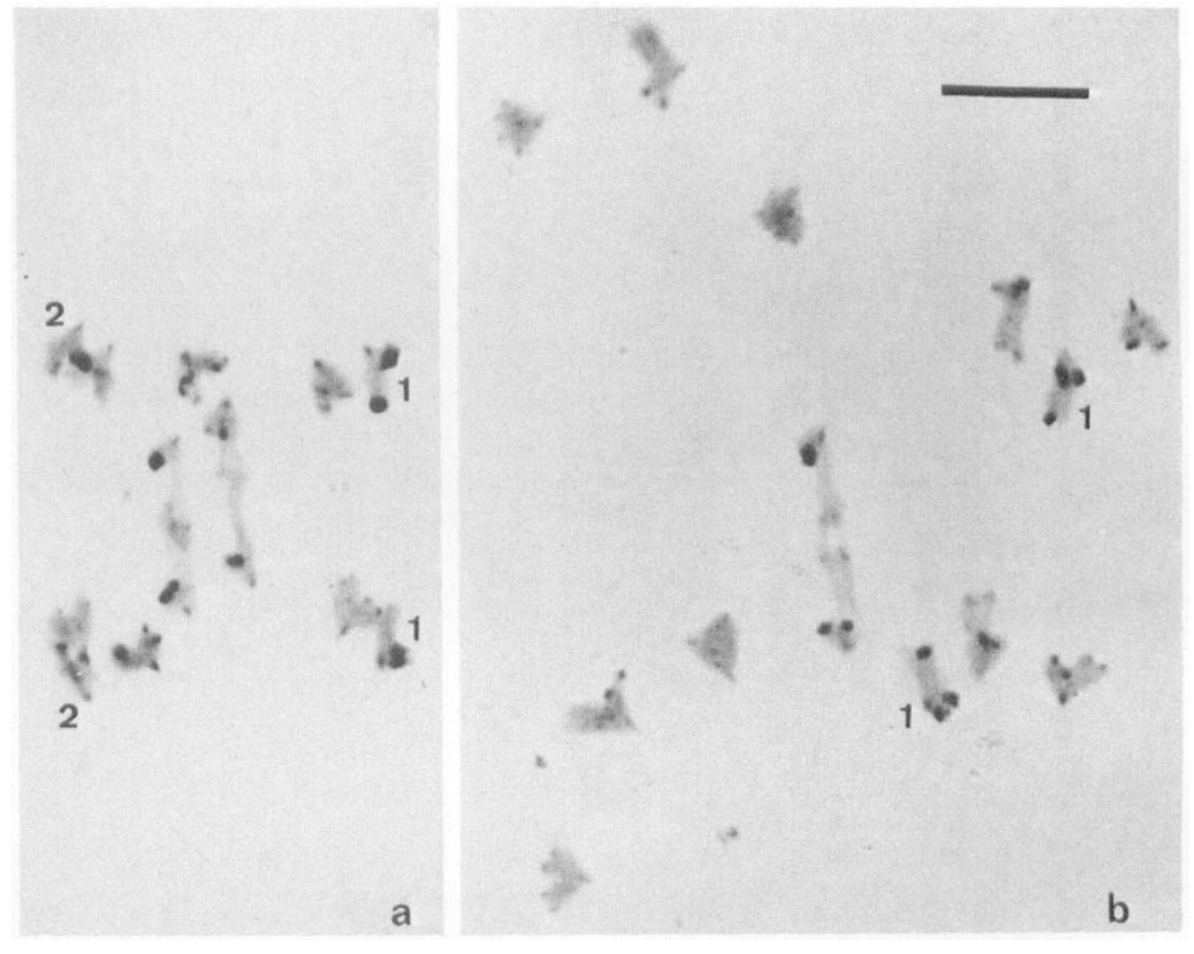

FIG. 2a-b. $C$-banded anaphase I cells. a, Backcross B1PEE (1R+-, 2R--). In this cell both chromosomes $1 R$ and $2 R$ are in parental form. b, Backcross B1PEE (1R+-, $2 R--)$. Recombinant type of chromosome $1 R$. Bar represents $10 \mu$. 

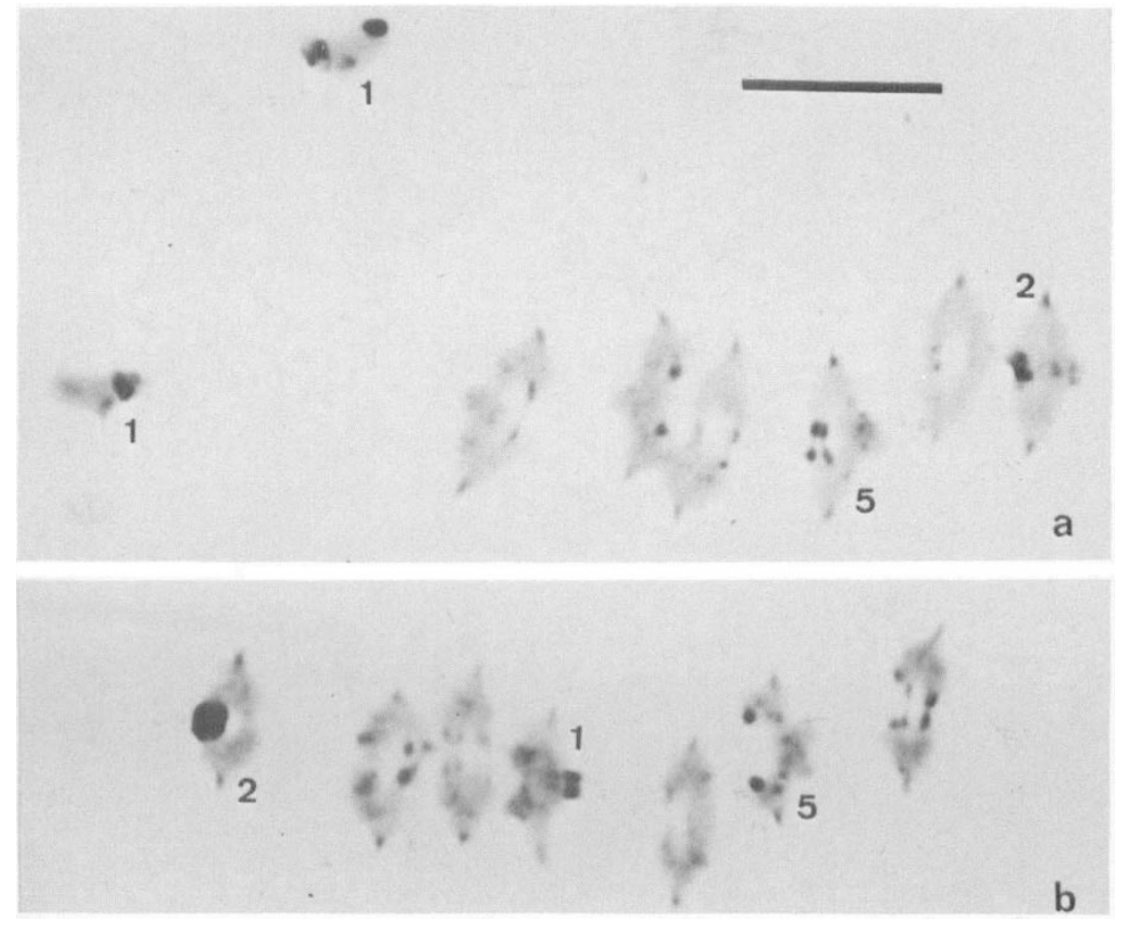

FIG. 1a-b. C-banded metaphase I cells. a, Plant of the backcross B1PEE $(1 \mathrm{R}+-, 2 \mathrm{R}+-)$ showing two univalents without evidence of recombination. $b$, Plant of the backcross B1PMM (1R+-, 2R++). The telomeric band in short arm of chromosome $2 \mathrm{R}$ of inbred line $M$ is specially big. Bar represents $10 \mu$. 
TABLE 1

Comparison between mean numbers of bound arms per cell at middle and late metaphase I in the different plants analyzed according to the C-heterochromatin constitution of chromosomes $1 \boldsymbol{R}$ (long arm) and $2 \boldsymbol{R}$ (short arm) (+-: chromosome heterozygous for presence of telomeric C-heterochromatin; - -: chromosome homozygous for absence of telomeric C-heterochromatin). 100 middle metaphase I cells and 100 late metaphase I cells per plant were observed. A paired $\mathrm{t}$ test was performed

\begin{tabular}{|c|c|c|c|c|c|c|}
\hline \multirow{4}{*}{$\begin{array}{c}\text { Mean of } \\
\text { bound } \\
\text { arms } \\
\text { per cell }\end{array}$} & \multicolumn{6}{|c|}{ C-heterochromatin constitution } \\
\hline & \multicolumn{2}{|c|}{$\begin{array}{c}1 \mathrm{R}(+-), 2 \mathrm{R}+-) \\
\quad(7 \text { plants })\end{array}$} & \multicolumn{2}{|c|}{$\begin{array}{c}1 \mathbf{R}(--), 2 \mathrm{R}(+-) \\
\quad(5 \text { plants })\end{array}$} & \multicolumn{2}{|c|}{$\begin{array}{l}1 \mathrm{R}(+-), 2 \mathrm{R}(--) \\
\quad(5 \text { plants })\end{array}$} \\
\hline & Middle M-I & Late $\mathbf{M}-\mathbf{I}$ & Middle M-I & Late M-I & Middle M-I & Late M-I \\
\hline & $12 \cdot 31$ & $11 \cdot 47$ & $12 \cdot 46$ & $11 \cdot 28$ & $12 \cdot 14$ & $10 \cdot 90$ \\
\hline$t$ & \multicolumn{2}{|c|}{$\begin{array}{c}5.6525 ; \text { d.f. }=6 \\
0.01>p>0.001\end{array}$} & \multicolumn{2}{|c|}{$\begin{array}{c}7.4136 ; \text { d.f. }=4 \\
0.01>p>0.001\end{array}$} & \multicolumn{2}{|c|}{$\begin{array}{c}9.1137 ; \text { d.f. }=4 \\
0.001>p\end{array}$} \\
\hline
\end{tabular}

\section{(i) Bound arm loss at metaphase I}

Plants of the backcross B1PEE having different C-banding patterns in chromosomes $1 \mathrm{R}$ and $2 \mathrm{R}$ were used in order to ascertain the existence of bound arm loss during metaphase I. In each plant the mean number of bound arms per cell and the probability of each identified bivalent arm being bound at metaphase I were scored in anthers in which all cells were at metaphase I (middle metaphase) and in anthers in which at least 50 per cent of the cells were in a later stage than metaphase I (late metaphase).

Table 1 shows the comparison between the mean numbers of bound arms per cell at middle and late metaphase $I$ in the different plants analyzed according to the $\mathrm{C}$-heterochromatin constitution of their chromosomes $1 \mathrm{R}$ and $2 \mathrm{R}$. In all cases, the number of bound arms per cell at middle metaphase is significantly higher than at late metaphase I.

Table 2 shows the comparison between the probabilities of each identified bivalent arm being bound at middle and late metaphase I. In all cases, the probability of being bound is higher at middle metaphase I than at late metaphase I, the differences being significant for the short arms of all bivalents.

These results agree with Orellana $e t$ al. (unpublished) that bound arm loss is taking place during metaphase I in desynaptic rye.

It is worth mentioning that if this bound arm loss during metaphase I were actually a loss of chiasmata the heterozygosity for telomeric teterochromatin shown by bivalents $1 R$ and $2 R$ would lead to the appearance of un-bound arms showing evidence of recombination, i.e., un-bound arms at metaphase I having telomeric $\mathrm{C}$-heterochromatin in only one of the two chromatids. Univalents or open bivalents at metaphase I showing evidence of recombination were not found.

\section{(ii) Chiasma frequency estimates}

As mentioned above, chromosomes $1 \mathrm{R}, 2 \mathrm{R}$ and $5 \mathrm{R}$ could be identified in the meiotic cells of all plants analyzed. In plants heterozygous for the 


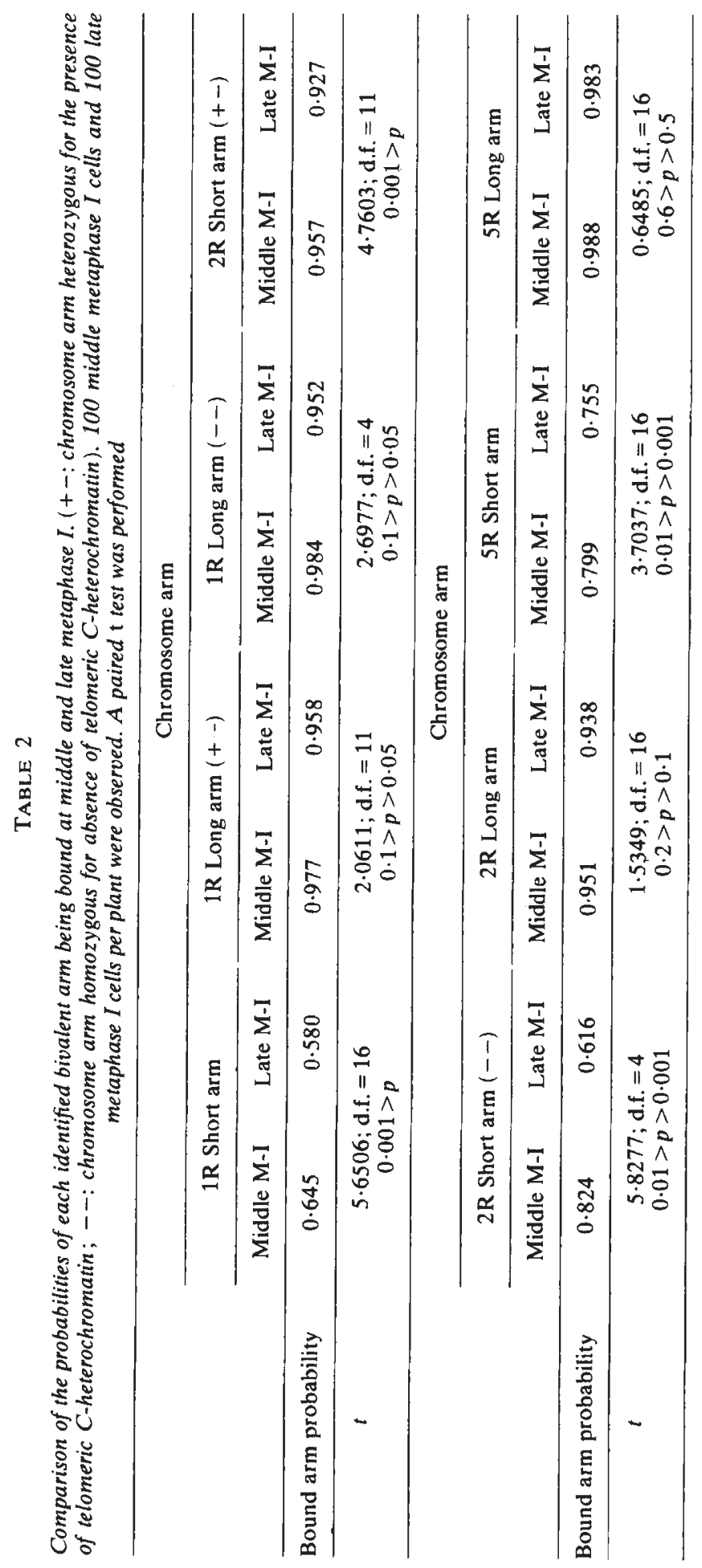


C-banding pattern of chromosomes $1 \mathrm{R}$ and $2 \mathrm{R}$, these chromosomes could show two different patterns at anaphase I: (i) Parental type: Both chromatids have the same $\mathrm{C}$-heterochromatin constitution showing no evidence of recombination. (ii) Recombinant type: Each chromatid has a different C-heterochromatin constitution showing evidence of recombination. Examples of these anaphase I chromosome types are shown in fig. 2.

If a bound arm corresponds to only one chiasma, the frequency of recombinant chromosomes in anaphase I must equal the frequency of bonds at metaphase I for a specific arm. However, an excesss of parental type chromosomes can appear at anaphase I if more than one chiasma per bond is formed.

Assuming that a maximum of two chiasmata were formed between the centromere and the C-heterochromatic band for which the two chromosomes are heterozygous and if there is no chromatid interference, the frequencies of recombinant $(\mathrm{Fr})$ and parental $(\mathrm{Fp})$ anaphase I chromosomes would be (Orellana and Giraldez, 1981):

$$
\mathrm{Fr}=f 1+\frac{1}{2} f 2 ; \quad \mathrm{Fp}=f 0+\frac{1}{2} f 2 ; \quad f 0+f 1+f 2=1 .
$$

In which $f 0, f 1$ and $f 2$ are the frequencies of 0,1 and 2 chiasmata respectively. As $f 0$ can be estimated from the metaphase I observations ( $f 0=$ frequency of being not bound at metaphase I), the frequencies of 1 and 2 chiasmata can be deduced.

Table 3 shows the frequency of the different middle metaphase I configurations (ring bivalents, $\mathbf{R}$; open bivalents in which the long arm was bound, 01; open bivalents in which the short arm was bound, Os; and univalent pairs, $U$ ) for chromosome $1 R$ as well as the frequencies with which it appears as parental $(\mathrm{Fp})$ and recombinant $(\mathrm{Fr})$ type at anaphase $\mathrm{I}$ in the plants heterozygous for the C-banding pattern of this chromosome.

In the comparison of metaphase I and anaphase I, results between plants variation can be observed. Although statistical tests can be made only for the totals and for a few individual plants, it can be concluded that there are some plants which fit the assumption of only one chiasma per bond while in most of them there is a significant excess of anaphase I parental-type chromosomes.

The estimates of $f 0, f 1$ and $f 2$, also included in table 3 , relate to the whole long arm of chromosome $1 \mathrm{R}$ since the C-heterochromatic band for which the two chromosomes are heterozygous is in the telomere of this arm.

Table 4 shows the frequency of the different middle metaphase I configurations for chromosome $2 \mathrm{R}$ as well as the frequencies of parental $(\mathrm{Fp})$ and recombinant $(\mathrm{Fr})$ anaphase I chromosomes in the plants heterozygous for a telomeric C-heterochromatic band in the short arm of this chromosome (Backcross B1PEE). If the assumption of only one chiasma per bond is made the excess of anaphase I parental-type chromosomes is significant in all plants except in two.

The anaphase data shown in tables 3 and 4 were obtained from 100 cells of only one anther per plant, except in plants $647 / 6,646 / 13$ and $647 / 22$ in which three anthers (100 cells each) were scored in order to determine the possible existence of between-anther variation for the number of recombinant and parental type anaphase I chromosomes. The contingence $\chi^{2}$ test indicated that there is no significant between-anther variation for these frequencies. 


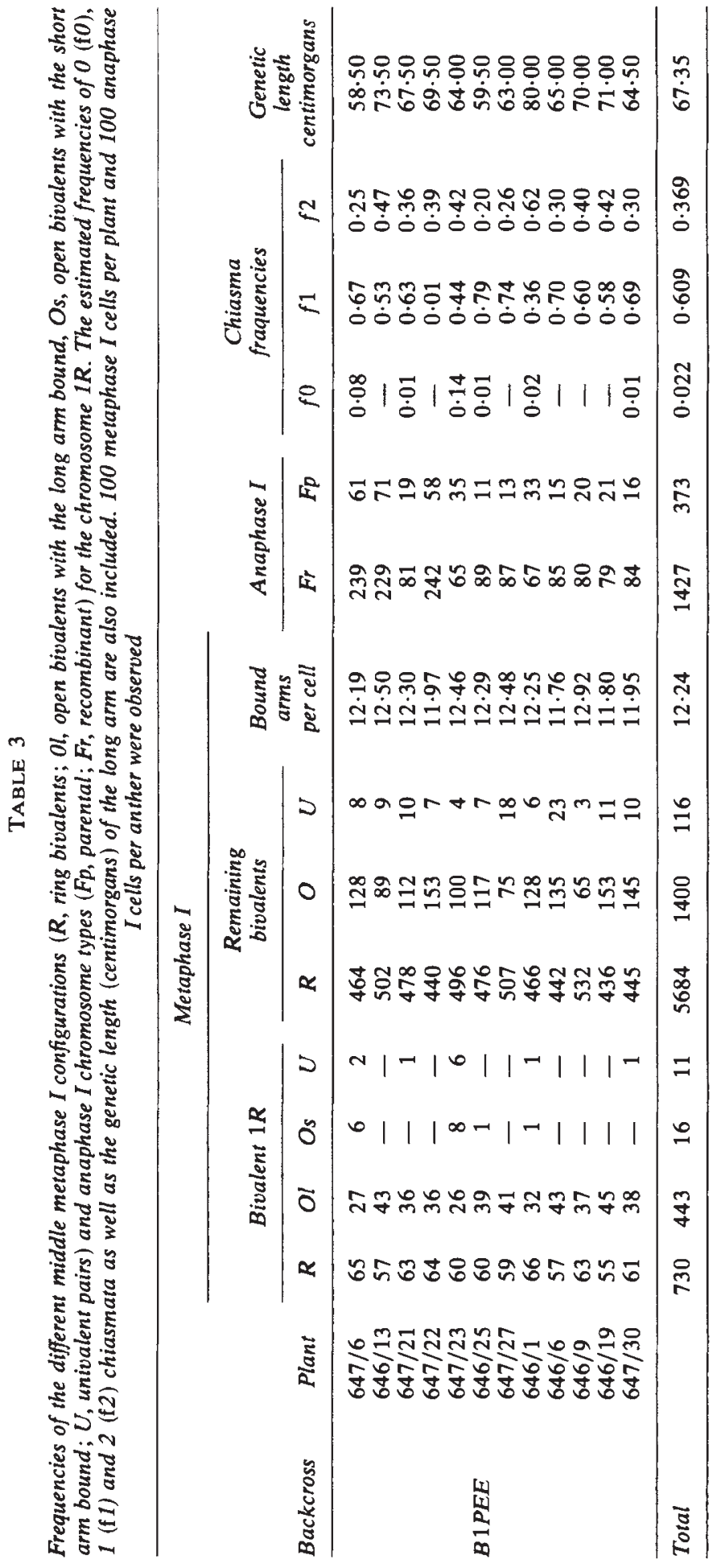




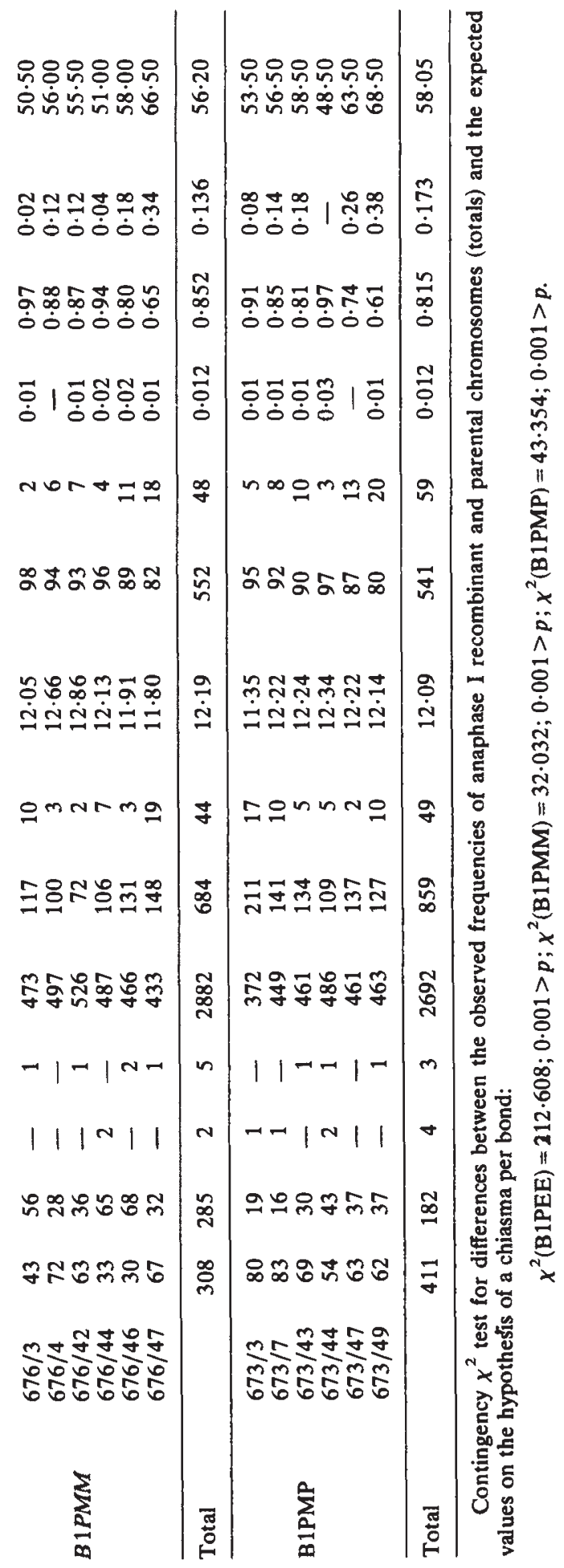



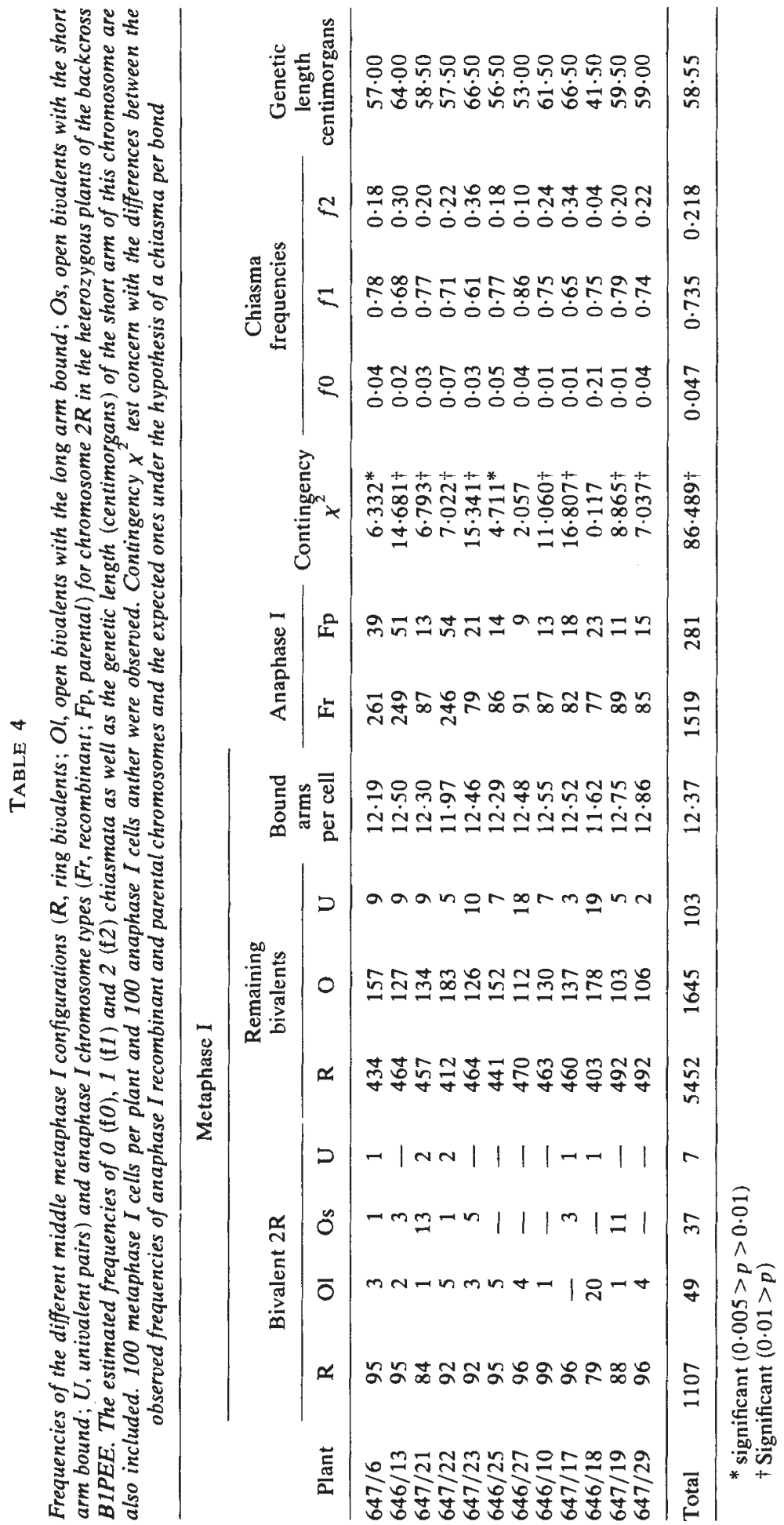


\section{Discussion}

It is a well-known fact that inbreeding in rye leads to a decrease in bound arm frequency and eventually to desynapsis. With this in mind, in the backcrosses studied in this work significant between-plant variation in bound arm frequency and chiasma frequency is expected, the values of such frequencies being no higher than those of the F1s from which they were produced.

Table 5 shows the bound arm frequencies at middle metaphase I, the frequencies of anaphase I recombinant and parental chromosomes and the estimated chiasma frequencies for chromosome arms $1 \mathrm{R}$ long and $2 \mathrm{R}$ short in the backcrosses studied in this work, compared with the corresponding values in the F1s from which the backcrosses were produced and with those in an open pollinated cultivar (Giraldez and Orellana, 1979; Orellana and Giraldez, 1981). From these comparisons it can be concluded:

(i) The probability of these chromosome arms being bound at middle metaphase I is similar in the backcrosses and in the corresponding F1s.

(ii) There is an excess of parental chromosomes at anaphase I in the backcross B1PEE when compared with F1PE. In most plants of this backcross the estimated chiasma frequencies of chromosome arms $1 \mathrm{R}$ and $2 \mathrm{R}$ short (Tables 3 and 4 ) are much higher than the ones of the corresponding $F 1$, being even higher than values obtained in the open pollinated cultivar Raña.

(iii) The estimated chiasma frequencies of backcross B1PMM and B1PMP are similar to those of F1PM. This is due to the between-plants variation found in these backcrosses (Table 3 ) in which unexpected plants with a higher chiasma frequency than that of the corresponding F1 are present.

Thus, most plants in the backcross B1PEE and some plants in backcrosses B1PMM and B1PMP behave in an unexpected way. The estimated chiasma frequencies in the chromosome arms analyzed are higher than that of the corresponding F1s.

Considering again the formulae from which the frequencies of $0(f 0)$, $1(f 1)$ and $2(f 2)$ chiasmata have been estimated (see section $3 \mathrm{ii}$ ) it can be concluded that the frequency of recombinant chromosomes reaches a maximum when $f 0=0$ and $f 2=0$ (i.e., when one and only one chiasma per arm is formed) and decreases either with a higher or a lower chiasma frequency. This is also true if the possibility of more than two chiasmata per arm is considered since, in that case (Mather, 1935):

$$
\begin{aligned}
& \mathrm{Fr}=f 1+\frac{1}{2} f 2+\frac{3}{4} f 3+\cdots+\frac{2}{3}\left[1-\left(-\frac{1}{2}\right)^{n}\right] f n \\
& \mathrm{Fp}=f 0+\frac{1}{2} f 2+\frac{1}{4} f 3+\cdots+\frac{1}{3}\left[1-\left(-\frac{1}{2}\right)^{n-1}\right] f n .
\end{aligned}
$$

The problem can then be enunciated in a different way. In these unexpected plants the frequency of recombinant chromosomes at anaphase I decreases as expected with a low chiasma frequency. However, the bound arm frequency at metaphase I does not correspond with such a decrease.

The simplest way to explain this discrepancy is to assume that there are some bonds which are not the result of at least one chiasma. One can imagine these non-chiasmate bonds as a remnant or prophase pairing. Examples of achiasmate organisms in which the chromosomes nevertheless remain paired until anaphase I onset are well known (see Baker et al., 1976). 


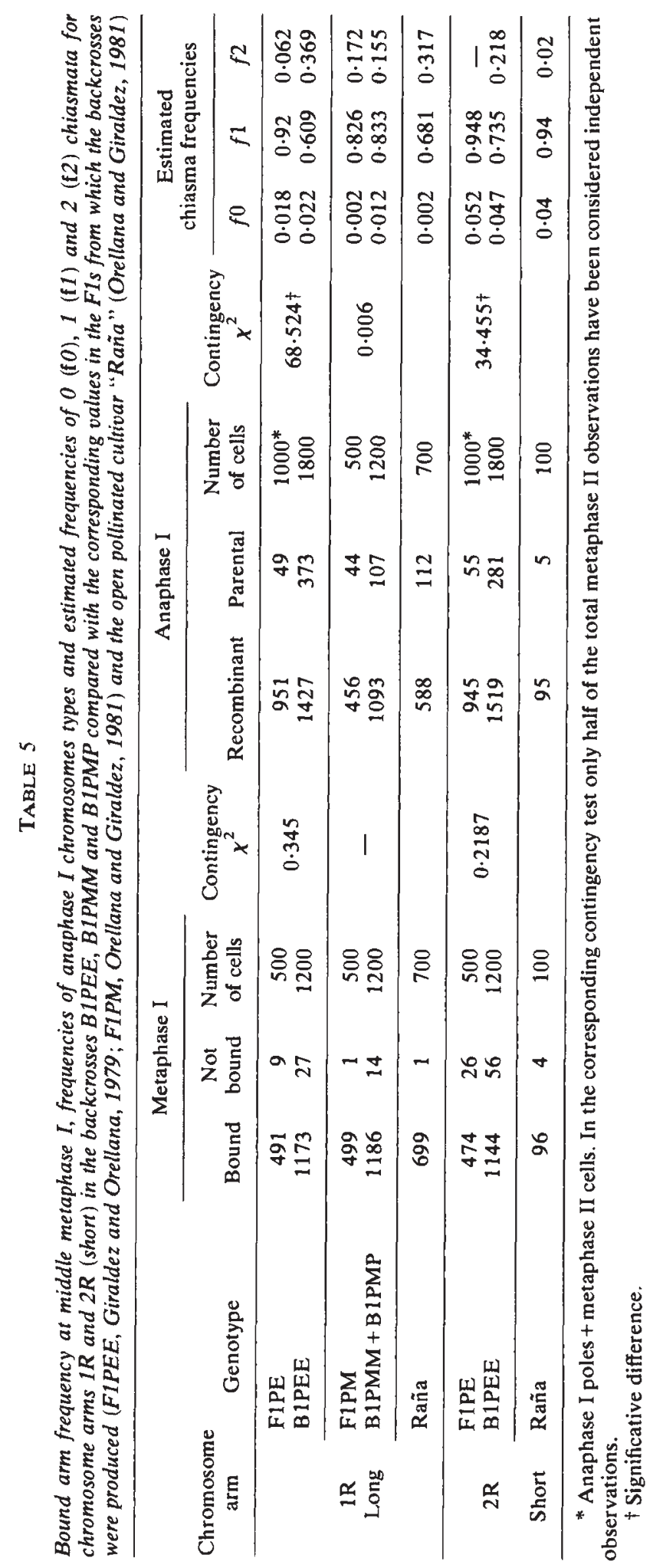


The C-heterochromatin bands in the chromosome arms analyzed are almost certainly telomeric. In these chromosome arms any chiasma formed would thus be located between the band and the centromere. The bound arm loss during metaphase I found in this material (Tables 1 and 2) together with the absence of univalents showing evidence of recombination (see fig. 1a) support the idea that the bonds lost are actually non-chiasmate.

The results obtained concerning the between plant variation for chiasma frequency in the backcrosses B1PEE, B1PMM and B1PMP can be explained on this point of view. The effect produced by these non-chiasmate bonds consists of a spurious increase in chiasma frequency, this effect being detected in plants in which chiasma frequency is lower than one chiasma per arm. In F1PE, chiasma frequency in the chromosome arms considered is very low (near one per arm as a maximum, Table 5). Thus, the decrease obtained in the plants of the backcross B1PEE (probably having less than one chiasma per arm) would produce the apparent increase in chiasma frequency (Table 3 and 4). On the other hand, F1PM has a higher chiasma frequency in chromosome arm $1 \mathrm{R}$ long (17 per cent double chiasmata, Table 5), some of the plants of the corresponding backcrosses would show a low decrease in chiasma frequency, the non-chiasmate bond effect not being apparent. In other plants of these backcrosses having a greater decrease in chiasma frequency, the false effect of chiasma frequency increase appears.

In chromosome arms in which a maximum of one chiasma is formed, the amount of non-chiasmate bonds (NCB) can be determined since:

$$
F p=N B+N C B \text {. }
$$

In which $\mathrm{Fp}$ is the frequency of parental chromosomes at anaphase I and NB is the frequency with which this arm is not bound at metaphase I. However, when the possibility of two chiasmata per arm is considered, the frequency of non-chiasmate bonds cannot be deduced:

$$
\begin{aligned}
& \mathrm{Fr}=f 1+\frac{1}{2} f 2 \\
& \mathrm{Fp}=\mathrm{NB}+\mathrm{NCB}+\frac{1}{2} f 2 .
\end{aligned}
$$

Now, considering in B1PEE a maximum of one chiasma per arm is probably occuring, the frequency of non-chiasmate bonds in chromosome arms $1 R$ (long) and $2 R$ (short) would be:

$$
\begin{aligned}
& \operatorname{NCB}(1 \mathrm{R} \text { long })=0.2384 \\
& \operatorname{NCB}(2 \mathrm{R} \text { short })=0 \cdot 1360 .
\end{aligned}
$$

This is in agreement with the results shown in table 2 in which a higher rate of bound arm loss can be observed in short arms. At middle metaphase I a higher number of non-chiasmate bonds have been lost in chromosome arm $2 \mathrm{R}$ (short), their frequency at that time being lower than that of chromosome arm $1 \mathrm{R}$ (long). Also, when the C-heterochromatin constitution of chromosome arm $2 \mathrm{R}$ short is considered (Table 2), a higher rate of bound arm loss in chromosome arms without telomeric C-heterochromatin (--) is observed. The results suggest that, in absence of chiasmata, maintenance of pairing at metaphase $\mathrm{I}$ is determined by arm length and telomeric $\mathrm{C}$-heterochromatin constitution. 
The results obtained in this work reveal the existence of a phenomenon in rye which can be compared to maintenance of pairing at metaphase I in achiasmatic organisms, suggesting the possibility of its existence in other organisms. Since this phenomenon is usually superimposed on chiasma formation, its detection is only possible in cases such as the one presented here.

It is worth mentioning the parallel that can be established between this work and that of Maguire (1978) in a desynaptic mutant of maize. This mutant had univalents at diakinesis that showed evidence of recombination (heterozygosity for heterochromatic knobs was used). She suggested that in that mutant the mechanism responsible for maintenance of pairing is altered but chiasma formation is not affected.

In the present work the effect of inbreeding in rye is the opposite to that observed by Maguire (1978) in the maize mutant. There is a decrease in chiasma frequency but maintenance of pairing seems not to be affected.

Acknowledgments. This work has been partially supported by a grant from the Comisión Asesora de Investigación Científica y Técnica of Spain.

\section{REFERENCES}

BAKER, B. S., CARPENTER, A. T. C., ESPOSITO, M. S., ESPOSITO, R. E. AND SANDLER, L. 1976. The genetic control of meiosis. Ann. Rev. Genet., 10, 53-134.

GIRALDEZ, R., CERMEÑO, M. C. AND ORELLANA, J. 1979. Comparison of C-binding pattern in the chromosomes of inbred lines and open pollinated varieties of rye. $Z$. Pflanzenzüchtg., 83, 40-48.

GIRALDEZ, R. AND ORElLANA, J. 1979. Metaphase I bonds, crossing over frequency, and genetic length of specific chromosome arms of rye. Chromosoma (Berl.), 72, 377-385.

MAGUIRE, M. P. 1978. Evidence for separated genetic control of crossing over and chiasma maintenance in maize. Chromosoma (Berl.), 65, 173-183.

MATHER, K. 1935. Reductional and equational separation of the chromosomes in bivalents and multivalents. $J$. Genet., 30, 53-78.

OREllaNA. J. AND GIRAlDEZ, R. 1981. Metaphase I bound arms and crossing over frequency in rye. I. Open pollinated varieties. Chromosoma (Berl.), 84, 439-449.

SYBENGA, J, 1982. Rye chromosome nomenclature and homoeolgy relationships. Workshop report. Z. Pflanzenzüchtg., (In press). 